\title{
J oint Kalman Channel Estimation and Turbo Equalization for MIMO OFDM Systems over Fast Fading Channels
}

\author{
Yu-Kuan Chang ${ }^{1}$, Fang-Biau Ueng ${ }^{1 *}$, Ye-Shun Shen ${ }^{2}$ and Chih-Yuan Liao ${ }^{1}$ \\ ${ }^{1}$ Department of Electrical Engineering \\ National Chung Hsing University \\ Taichung, Taiwan \\ Email: fbueng@nchu.edu.tw \\ ${ }^{2}$ Department of Aeronautical Engineering \\ National Formosa University \\ Yunlin, Taiwan \\ Email: ysshen@nfu.edu.tw \\ *Corresponding author: Fang-Biau Ueng
}

Received April 11, 2018; revised August 7, 2018; revised April 9, 2019; accepted May 30, 2019; published November 30, 2019

\begin{abstract}
The paper investigates a novel detector receiver with Kalman channel information estimator and iterative channel response equalization for MIMO (multi-input multi-output) OFDM (orthogonal frequency division multiplexing) communication systems in fast multipath fading environments. The performances of the existing linear equalizers (LE) are not good enough over most fast fading multipath channels. The existing adaptive equalizer with decision feedback structure (ADFE) can improve the performance of LE. But error-propagation effect seriously degrades the system performance of the ADFE, especially when operated in fast multipath fading environments. By considering the Kalman channel impulse response estimation for the fast fading multipath channels based on CE-BEM (complex exponential basis expansion) model, the paper proposes the iterative receiver with soft decision feedback equalization (SDFE) structure in the fast multipath fading environments. The proposed SDFE detector receiver combats the error-propagation effect for fast multipath fading channels and outperform the existing LE and ADFE. We demonstrate several simulations to confirm the ability of the proposed iterative receiver over the existing receivers.
\end{abstract}

Keywords: Kalman channel estimation, SDFE, turbo channel equalizer 


\section{Introduction}

The advanced wireless systems provides multi-media services that requires very high data rates. By configuring several antennas configuration at both the receiver and the transmitter, we can achieve high spectral efficiency. By inserting the cyclic prefix (CP), the OFDM technique can be used to effectively combat the multipath fading channel effect. The OFDM can changes the time-domain multipath fading channel effect into non-selective frequency fading. So the communication receiver design can be very simple to implement. The MIMO-OFDM communication systems can be employed to provide high data rates, and the design of the receiver can be very simple. So the MIMO-OFDM scheme are very popular in recent communication systems [1].

Channel impulse response estimations were widely spreadly employed for turbo equalizer design to predict the channel impulsse response cofficients of the time-varying fast multipath fading environments. The Kalman filter channel impulse response predictor can be employed for vehicular communications [2] [3] to effectively predict the fast multipath fading channels. By employing the channel estimation of the AR-1 channel, we can track the complex-exponential basis expansion models (CE-BEMs) channel state parameters [4] [5]. The Kalman filter channel impulse response predictor is an effective and low-cost channel response predictor. The estimator is with optimal bit error rate performance. In [6], the author provided an iterative receiver and can be used in MIMO fading multipath channels. However, the proposed scheme only employs Kalman estimation based on the previous hard decision which is produced by the minimum mean-square-error decision feedback equalization. In [7], the multipath fading environment is modeled as an AR system model and employs the modified KF channel estimation to predict the AR channel coefficients. In [8], several blind channel impulse response predictor of the multicarrier system is employed for the multi-input multi-output multipath fading channels. But the channel state information is assume to be constant, not fast multipath fading channels.

In multipath fading channels, the MIMO received signal is corrupted by coantenna interference, intersymbol interference (ISI) and spatial interference. Thus, the probability of doing an decision error is very high. The effect can result in very serious error propagation. Turbo equalizer [9] [10] with joint decoding and equalization can be employed in the advanced wireless communications.

Soft feedback equalizer (SFE) was investigated in [11]. The SFE coefficients can be computed so as to minimize the MSE (mean-squared-error). The structure [12] is also employed in underwater-acoustic system [13]-[17] and is employed in the multiinput multioutput systems. In this paper, a novel Kalman channel impulse response estimator and minimum-mean-square-error-based MIMO-OFDM SDFE is proposed. The coefficients of the soft-decision-feedback-equalizer are then derived based on the soft decisions of the data symbols.

\section{System Model}

\subsection{MIMO OFDM SDFE Transmitter}

The Fig. 1 is the MIMO OFDM SDFE transmitter, 


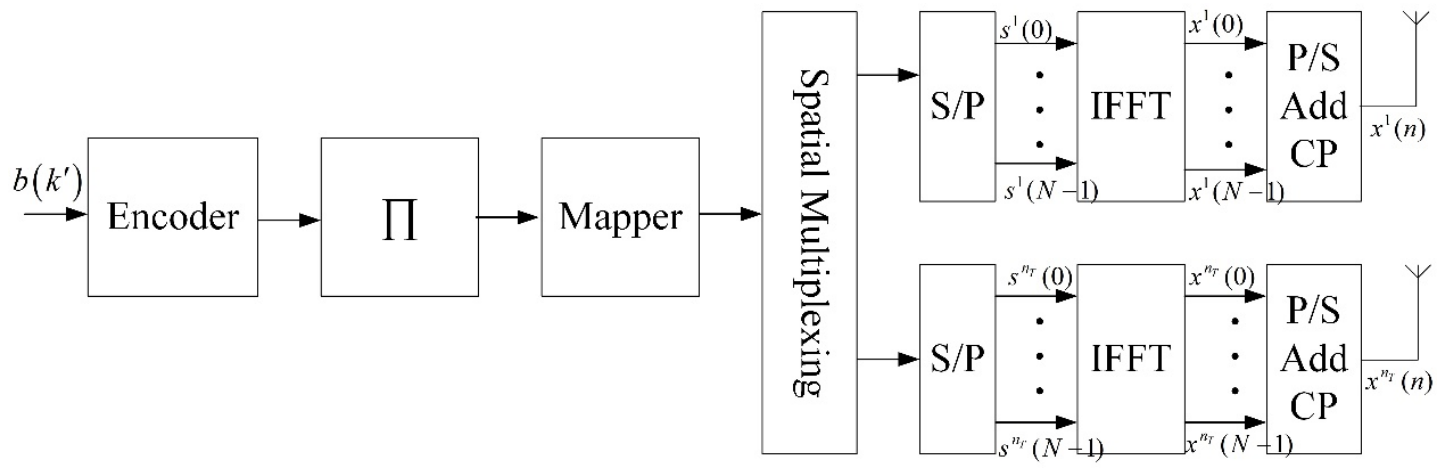

Fig. 1. The MIMO-OFDM SDFE transmitter.

The MIMO OFDM wireless communication is with $N$ frequency subcarriers, $n_{T}$ transmitting antennas, and $n_{R}$ receiver antennas, and equipped with digital modulation technique which is with modulation order $M=2^{m}$. The input data signal sequence $b\left(k^{\prime}\right)$ is then first digital encoded employing a recursive systematic convolutional (RSC). The recursive systematic convolutional digital encoder provides the digital channel correction coded symbols and are then next by the interleaver $\Pi$, and then followed by the modulation mapper. The symbol interleaver is employed to improve the system performance that the data don't suffer from burst-error and in order for the goal of turbo equalizer that will have uncorrelated data extrinsic symbol information. The output information signal of the modulation mapper is described as $S^{t}(k)$. The transmitted data symbols are selected from the i.i.d. samples. The transmitting power to be used is assumed to be equal to unit power, then the data alphabet is the $2^{m}$ - ary $Q=\left[\begin{array}{llll}\alpha_{1} & \alpha_{2} & \cdots & \alpha_{2^{m}}\end{array}\right]$, where $\alpha_{i}$ is the bit pattern $\mathbf{q}_{i}=$ $\left[\begin{array}{lll}q_{i, 1} & \ldots & q_{i, m}\end{array}\right]$ with $q_{i, m} \in 0,1$. We assume that the alphabet are with zero mean $\sum_{i=1}^{2^{m}} \alpha_{i}=0$ and unit energy $\frac{1}{2^{m}} \sum_{i=1}^{2^{m}}\left|\alpha_{i}\right|^{2}=1$.

After the modulation mapper, the spatial multiplexer is employed to separate the transmitted signal into $n_{T}$ streams. For orthogonal frequency division multiplexing transmission, the data symbol block usess a $\mathrm{S} / \mathrm{P}$ converter which is with $N$ symbols. Then, employing $N$-point IFFT, each data symbol block is then changed into the time-domain data sequence. A cyclic prefix (CP) which is with length $v$ is then appeded. The transmitting symbol signal $x^{t}(n)$ for the $t-t h$ transmitted antenna then be denoted as

$$
x^{t}(n)=\frac{1}{\sqrt{N}} \sum_{k=0}^{N-1} s^{t}(k) e^{j 2 \pi r k / N},
$$

The received data signals in any of the $r$ - th receiving antennas in base band can be described as follows,

$$
y^{r}(n)=\sum_{t=1}^{n_{t}} \sum_{l=0}^{L-1} \hat{h}^{r, t}(t ; l) x^{t}(n-l)+v_{r}(n)
$$

where $\hat{h}^{r, t}(t ; l)$ denotes is the $l-t h$ gain of the channel impulse response corresponding to the $t-t h$ transmitting antenna and the $r-t h$ receiving antenna at time $t, v_{r}(n)$ is the AWGN noise sample with zero mean and variance $\sigma_{w}^{2}$ on the $r-t h$ receiving antenna. 


\subsection{The Proposed Multi-Input Multi-Output SDFE Receiver Structure}

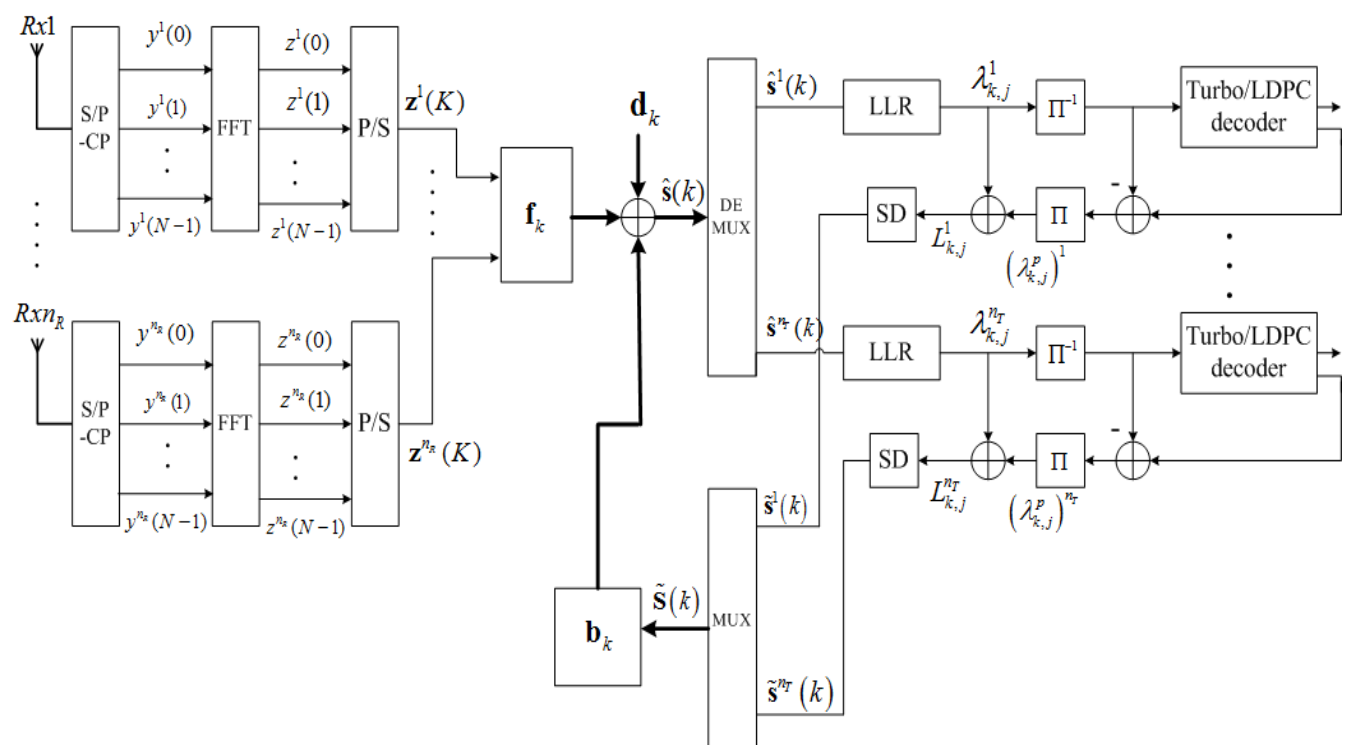

Fig. 2. The MIMO-OFDM SDFE receiver.

The received sample $y^{r}(n)$ is collected employing a $\mathrm{S} / \mathrm{P}$ converter. In the received antenna $r$, we apply FFT for the received time-domain signal samples when we remove the CP, and then yields a frequency-domain signal sample vector. We write the received signal vector in matrix form as the following,

$$
\boldsymbol{z}^{r}=\sum_{t=1}^{n_{t}} \mathbf{F} \widehat{\mathbf{H}}^{r, t} \mathbf{F}^{H} S^{t}+w^{r},
$$

where the $\mathbf{F}$ represents the unitary FFT, $\widehat{\mathbf{H}}^{r, t}$ denotes the channel matrix corresponding to the $t-t h$ transmitting antenna and the $r-t h$ receiving antenna, the $\mathbf{z}^{r}$ is the received signal symbol vector, the $\mathbf{s}^{t}$ is the transmitting signal symbol vector, the $w^{r}$ is the noise vector. The $\mathbf{z}^{r}$ and $w^{r}$ can be described as following,

$$
\begin{array}{lll}
\boldsymbol{z}^{r}=\left[\begin{array}{lll}
z^{r}(0) & \cdots & z^{r}(N-1)
\end{array}\right]^{T} \\
w^{r}=\left[\begin{array}{lll}
w^{r}(0) & \cdots & w^{r}(N-1)
\end{array}\right]^{T}
\end{array}
$$

The block diagram of the proposed iterative receiver of the MIMO-OFDM-SDFE can be shown as Fig. 2. The propsoed low-complexity iterative frequecny-domain receiver is design based on individual frequency. So, the frequency sampling signal used to explore the signals that is for combination as the following equation[18],

$$
\mathbf{Z}(k)=\widehat{\mathbf{H}}(\mathrm{k}) \mathbf{S}(k)+W(k)
$$

where we define the matrices $\mathbf{Z}(k) \in C^{n_{r} \times 1}, \widehat{\mathbf{H}}(k) \in C^{n_{t} \times n_{r}}, \mathbf{S}(k) \in C^{n_{t} \times 1}$ and $W(k) \in$ $C^{n_{r} \times 1}$ as follows,

$$
\begin{aligned}
& \mathbf{Z}(k)=\left[\begin{array}{lll}
z^{1}(k) & \cdots & z^{N_{r}}(k)
\end{array}\right]^{T} \\
& \widehat{\mathbf{H}}=\left[\begin{array}{ccc}
\hat{\bar{g}}_{1,1}(k) & \cdots & \hat{\bar{g}}_{1, n_{t}}(k) \\
\vdots & \ddots & \vdots \\
\hat{\bar{g}}_{n_{r}, 1}(k) & \cdots & \hat{\bar{g}}_{n_{r}, n_{t}}(k)
\end{array}\right] \\
& \mathbf{S}(k)=\left[\begin{array}{lll}
s^{1}(k) & \cdots & s^{N_{t}}(k)
\end{array}\right] \\
& W(k)=\left[\begin{array}{lll}
w^{1}(k) & \cdots & w^{N_{t}}(k)
\end{array}\right]
\end{aligned}
$$


The employed SDFE turbo equalizer is constructed by a feedback filter and a feed forward filter. The feedback filterand the feed forward filter are employed for all the subcarriers. The MMSE signal estimator $\hat{S}(k)$ of the transmitting signal symbol for the $k-t h$ frequency subcarrier can be described as follows,

$$
\begin{gathered}
\widehat{\mathbf{S}}(k)=\mathbf{f}_{k} \mathbf{Z}(k)+\mathbf{b}_{k} \tilde{\mathbf{S}}(k)+\mathbf{d}_{k} \\
\tilde{\mathbf{S}}(k)=\left[\begin{array}{llll}
\tilde{s}^{1}(k) & \tilde{s}^{2}(k) & \cdots & \tilde{s}^{N_{t}}(k)
\end{array}\right]
\end{gathered}
$$

where the $\tilde{\mathbf{S}}(k)$ denotes the past decided signal estimator of the $k-t h$ subcarrier in the $t-t h$ receiving antenna. An offset compensation signal $\mathbf{d}_{k}$ is employed to achieve the reliability of both soft decisions and soft a priori information of the transmitted data signal symbols. In order to design the proposed iterative receiver [19] [20], the soft decision feedback equalizer output signal at the $k-t h$ frequency subcarrier in (11) can be derived by performing the minimization of the mean square error as follows,

To obtain the coefficients $\mathbf{f}_{k}, \mathbf{b}_{k}$ and $\mathbf{d}_{k}$,

$$
\varepsilon_{k}^{2}=E\left\{|\hat{\boldsymbol{s}}(k)-\boldsymbol{s}(k)|^{2}\right\}
$$

We then obtain

$$
\frac{\partial \varepsilon_{k}^{2}}{\partial \boldsymbol{d}_{k}^{*}}=-2 E\left\{\mathbf{s}(k)-\mathbf{f}_{k} \mathbf{Z}(k)-\mathbf{b}_{k} \tilde{\mathbf{s}}(k)-\mathbf{d}_{k}\right\}=0
$$

$$
\mathbf{d}_{k}=E\{\boldsymbol{s}(k)\}-\mathbf{f}_{k} \widehat{\mathbf{H}} E\{\mathbf{S}(k)\}-\mathbf{b}_{k} E\{\tilde{\mathbf{S}}(k)\}
$$

So the optimal feedback and the feed forward coefficients can be computed based on the following two equations,

Then

$$
\begin{aligned}
& \frac{\partial \varepsilon_{k}^{2}}{\partial \mathbf{f}_{k}^{H}}=-2 E\left\{\left(\mathbf{s}(k)-\mathbf{f}_{k} \mathbf{Z}(k)-\mathbf{b}_{k} \tilde{\mathbf{s}}(k)-\mathbf{d}_{k}\right) \mathbf{Z}^{H}(k)\right\}=0 \\
& \frac{\partial \varepsilon_{k}^{2}}{\partial \mathbf{b}_{k}^{H}}=-2 E\left\{\left(\mathbf{s}(k)-\mathbf{f}_{k} \mathbf{Z}(k)-\mathbf{b}_{k} \tilde{\mathbf{s}}(k)-\mathbf{d}_{k}\right) \tilde{\mathbf{S}}^{H}(k)\right\}=0
\end{aligned}
$$

So

$$
\begin{gathered}
\boldsymbol{e}_{k}=\left(\sigma_{w}^{2} \boldsymbol{I}_{N n_{r}}+\widehat{\mathbf{H}} \mathbf{R}_{k}^{f f} \widehat{\mathbf{H}}^{H}\right) \mathbf{f}_{k}^{H}+\widehat{\mathbf{H}} \mathbf{R}_{k}^{f b} \mathbf{b}_{k}^{H} \\
\mathbf{f}_{k} \widehat{\mathbf{H}} \mathbf{R}_{k}^{f b}+\mathbf{b}_{k} \mathbf{R}_{k}^{f b}=0
\end{gathered}
$$

$$
\begin{gathered}
\mathbf{f}_{k}^{H}=\left[\sigma_{w}^{2} \boldsymbol{I}_{N n_{r}}+\widehat{\mathbf{H}}\left(\mathbf{R}_{k}^{f f}-\mathbf{R}_{k}^{f b}\left(\mathbf{R}_{k}^{b b}\right)^{-1} \mathbf{R}_{k}^{f b H}\right) \widehat{\mathbf{H}}^{H}\right]^{-1} \mathbf{e}_{k} \\
\mathbf{b}_{k}^{H}=-\left(\mathbf{R}_{k}^{b b}\right)^{-1}\left(\widehat{\mathbf{H}} \mathbf{R}_{k}^{f b}\right)^{H} \mathbf{f}_{k}^{H}
\end{gathered}
$$

where the covariance matrices $\mathbf{R}_{k}^{f f} \in C^{N n_{T} \times N n_{T}}, \mathbf{R}_{k}^{f b} \in C^{N n_{T} \times N n_{T}}, \mathbf{R}_{k}^{b b} \in C^{N n_{T} \times N n_{T}}$ and $\mathbf{e}_{k} \in C^{N n_{R} \times N n_{T}}$ and are described as the following equations,

$$
\begin{aligned}
& \mathbf{R}_{k}^{f f}=E\left\{\mathbf{S}(k) * \mathbf{S}(k)^{H}\right\}-E\{\mathbf{S}(k)\} * E\left\{\mathbf{S}(k)^{H}\right\} \\
& \mathbf{R}_{k}^{f b}=E\left\{\mathbf{S}(k) * \tilde{\mathbf{S}}(k)^{H}\right\}-E\{\mathbf{S}(k)\} * E\left\{\tilde{\mathbf{S}}(k)^{H}\right\} \\
& \mathbf{R}_{k}^{b b}=E\left\{\tilde{\mathbf{S}}(k) * \tilde{\mathbf{S}}(k)^{H}\right\}-E\{\tilde{\mathbf{S}}(k)\} * E\left\{\mathbf{S}(k)^{H}\right\} \\
& \mathbf{e}_{k}=\mathbf{H}\left(E\left\{\mathbf{S}(k) * \mathbf{S}(k)^{H}\right\}-E\{\mathbf{S}(k)\} * E\left\{\mathbf{s}(k)^{H}\right\}\right)
\end{aligned}
$$

For the output of the proposed iterative detector, assuming that the estimate signal vector $\hat{\mathbf{s}}(k)$ as the output signal of an equivalent AWGN channel. So we can use the additive white Gaussian noise model to represent the extrinsic LLR (log likelihood ratios) $\lambda_{k}^{t}$. Based on another equivalent AWGN channel model, we can also do the computation to get the a priori $\log$ likelihood ratios (LLR) $\left(\lambda_{k}^{t}\right)^{p}$. Here, the superscript ()$^{p}$ represents the quantities of the signal provided by the previous iteration of the proposed receiver. Next, we add the a priori 
information $\left(\lambda_{k}^{t}\right)^{p}$ to the extrinsic information $\lambda_{k}^{t}$ to obtain the full LLR $L_{k}^{t}$. The $L_{k}^{t}$ is used the computation of the estimation $\tilde{s}(k)$ and then the covariance matrices of the signal vector.

\section{The Proposed Iterative SDFE Receiver}

For the $k-t h$ frequency subcarrier, the signal estimator $\widehat{\mathbf{s}}(k)$ is described as follows,

$$
\widehat{\mathbf{s}}(k) .=\mathbf{f}_{k}(\mathbf{Z}(k)-\widehat{\mathbf{H}} E\{\mathbf{S}(k)\})+\mathbf{b}_{k}(\tilde{\mathbf{S}}(k)-E\{\tilde{\mathbf{S}}(k)\})+E\{\mathbf{s}(k)\}
$$

The proposed iterative SDFE receiver can be described as the Fig. 3. So $E\{\mathbf{s}(k)\}=0$, $E\{\tilde{\mathbf{S}}(k)\}=0$, the proposed soft decision feedback equalizer can be regarded as an traditional DFE. The coefficients of the feedforward filter can be represented as

$$
\mathbf{f}_{k}^{H(1)}=\left[\sigma_{w}^{2} \mathbf{I}_{N n_{r}}+\widehat{\mathbf{H}} \widehat{\mathbf{H}}^{H}\right]^{-1} \boldsymbol{e}_{k}
$$

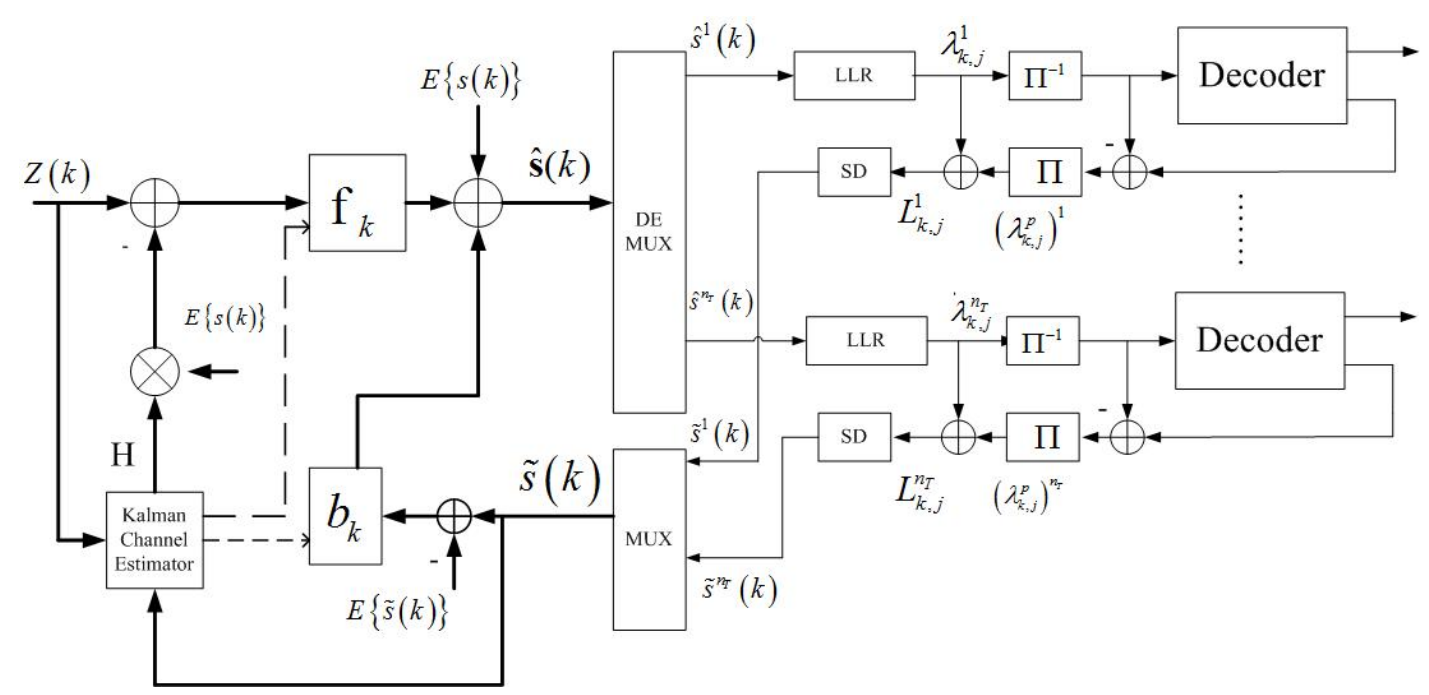

Fig. 3. The proposed SDFE receiver.

For the computations of the $\mathbf{f}_{k}, \mathbf{b}_{k}$ and $\mathbf{d}_{k}$, we must compute the $\mathbf{R}_{k}^{f f}, \mathbf{R}_{k}^{f b}$ and $\mathbf{R}_{k}^{b b}$. The $\mathbf{R}_{k}^{f f}, \mathbf{R}_{k}^{f b}$ and $\mathbf{R}_{k}^{b b}$ are very important to derive the signal estimator $\widehat{\mathbf{s}}(k)$. Based on the above equations (22), (23) and (24), the $\mathbf{R}_{k}^{f f}$ can be determined [15]-[19]. For calculating $\mathbf{R}_{k}^{f b}$ and $\mathbf{R}_{k}^{b b}$, based on the assumption that the transmitting signal symbols are i.i.d., so we can argue that $E\left\{s^{p}(k) * \tilde{s}^{q}(k)\right\}=E\left\{\tilde{s}^{p}(k) * \tilde{s}^{q}(k)\right\}=0$. We also simplify the computation as values $\varepsilon^{t}, \chi^{t}$ and $\xi^{t}$ that are represented as follows [15],

$$
\begin{gathered}
\varepsilon^{t}=E\left\{\tilde{s}^{t}(k)\right\} \\
\chi^{t}=E\left\{s^{t}(k) * \tilde{s}^{t}(k)^{*}\right\} \\
\xi^{t}=E\left\{\tilde{s}^{t}(k) * \tilde{s}^{t}(k)^{*}\right\}
\end{gathered}
$$

However, these individual expectation values $\varepsilon^{t}, \chi^{t}$ and $\xi^{t}$ have to be decided based on the data samples. Since the $\varepsilon^{t}, \chi^{t}$ and $\xi^{t}$ are the functions of the signals $\tilde{\mathbf{s}}(k)$, and the signals $\tilde{\mathbf{s}}(k)$ are the function of $\left(\lambda_{k}^{t}\right)^{p}$ and the extrinsic LLR $\lambda_{k, j}^{t}$. So, knowledge of $\left(\lambda_{k}^{t}\right)^{p}$ and $\lambda_{k}^{t}$ is necessary to derive the signal matrix. The receiver flowchart of the iterative receiver scheme is described as the Fig. 4. At first, the pilot signal is sent to get the channel estimation $\widehat{H}$. Then 
the receiving signal vector $\mathbf{Z}(k)$ goes through the filter $\mathbf{f}_{k}^{H(1)}$. In the first iteration of the receiver, (27) can be employed. We then obtained the estimation of the signal symbols $\hat{s}^{t}(k)$. For the equalizer output, assuming that the output signal is derived from an equivalent AWGN channel, and can be used to compute $\lambda_{k, j}^{t}$ [21]- [25], we also get the a priori log likelihood ratios (LLR) $\left(\lambda_{k}^{t}\right)^{p}$. Adding $\lambda_{k}^{t}$ to $\operatorname{LLR}\left(\lambda_{k}^{t}\right)^{p}$ to obtain the full LLR $L_{k}^{t}$. And then, the full LRR $L_{k}^{t}$ passes the soft decision of the receiver and then used to calculate $p\left(\tilde{s}^{t}(k)=\alpha_{i}\right)$. So the $p\left(\tilde{s}^{t}(k)=\alpha_{i}\right)$ can be used to calculate the past decided estimation signal $\tilde{\mathbf{s}}(k)$. Since $\varepsilon^{t}$, $\chi^{t}$ and $\xi^{t}$ are the functions of the signal $\tilde{\mathbf{s}}(k)$, so the $\varepsilon^{t}, \chi^{t}$ and $\xi^{t}$ can be derived by the previous determined estimation signal $\widetilde{\mathbf{s}}(k)$. Finally, the $\varepsilon^{t}, \chi^{t}$ and $\xi^{t}$ can be employed to derive the $\mathbf{R}_{k}^{f b}$ and $\mathbf{R}_{k}^{b b}$.

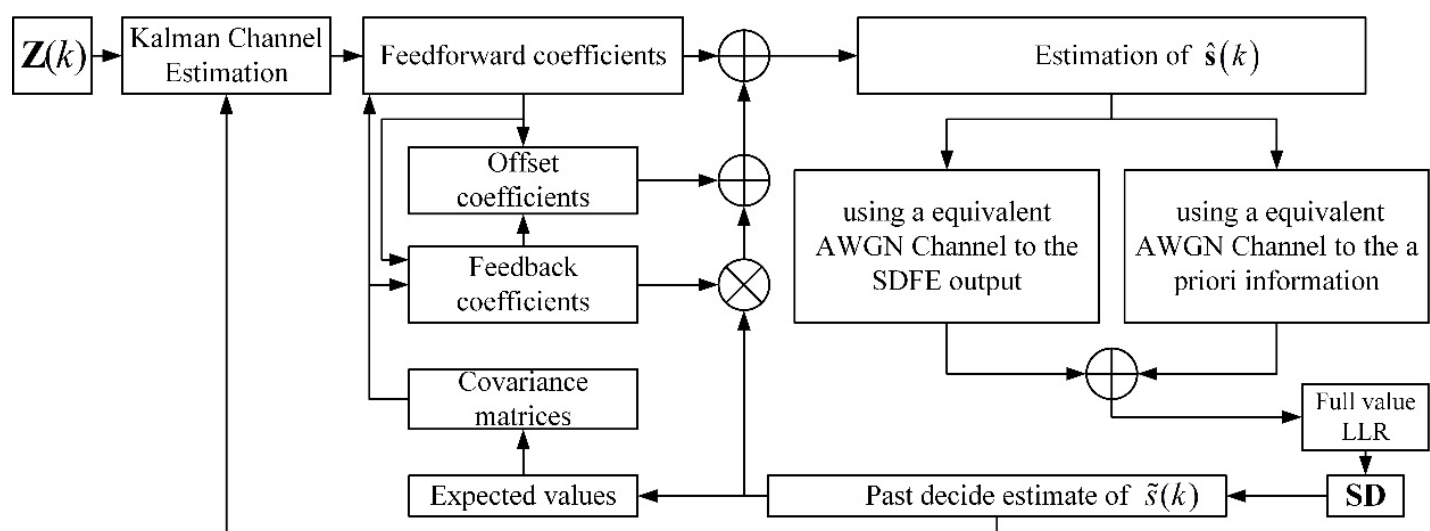

Fig. 4. The flowchart of the proposed scheme.

\section{Kalman Estimation}

The vector of CE-BEM parameters are

$$
\begin{gathered}
\bar{h}_{r, t}(k)=\left[\square_{r, t, 1}(k ; 0), \cdots, \square_{r, t, Q}(k ; 0), \square_{r, t, 1}(k ; L-1), \cdots, \square_{r, t, Q}(k ; L-1)\right] \\
\bar{g}_{r, t}(k)=\mathbf{B}(k) * \square_{r, t}(k)
\end{gathered}
$$

where $r=1, \ldots, n_{r}, t=1, \ldots, n_{t}$, and

$$
\begin{gathered}
\mathbf{B}(k):=\mathbf{I}_{L+1} \otimes \bar{b}_{e x}(k) \\
\bar{b}_{e x}(k):=\left[e^{j w_{1} n}, e^{j w_{2} n}, \ldots, e^{j w_{Q} n}\right] .
\end{gathered}
$$

The CE functions get the fast variation of the communication channel. The evolution of the complex-exponential basis expansion model coefficients are assumed to be an AR-1 model,

$$
\mathbf{h}_{a, r, t}(k)=\alpha * \mathbf{h}_{a, r, t}(k-1)+\bar{w}(k)
$$

The dynamic model for the fixed-lag $\mathrm{KF}$ is as

$$
\begin{aligned}
\mathbf{h}_{a, r, t}(k) \approx\left[\square_{r, t}(k), \square_{r, t}(k-1), \ldots, \square_{r, t}(k-D)\right]^{T} \\
\triangleq\left[\square_{a, r, t, 0}(k) \square_{a, r, t, 1}(k) \ldots \square_{a, r, t, D}(k)\right]^{T}
\end{aligned}
$$

The measurement and the state equations for the Kalman filter are as

In addition, we have

$$
\begin{gathered}
\mathbf{h}_{a, r, t}(k)=\mathbf{F}_{a, r, t}(k) * \mathbf{h}_{a, r, t}(k-1)+\mathbf{G}_{a, r, t}(k) * \mathbf{w}(k) \\
\mathbf{y}(k)=\mathbf{E}_{a, r, t}(k) * \mathbf{h}_{a, r, t}(k)+v(k)
\end{gathered}
$$


and

$$
\begin{aligned}
& \mathbf{F}_{a, r, t}(k)=\left[\begin{array}{cccc}
\alpha \mathbf{I}_{Q(L+1)} & 0 & \ldots & 0 \\
\mathbf{I}_{Q(L+1)} & 0 & \ldots & 0 \\
\vdots & \vdots & \ddots & \vdots \\
0 & 0 & \mathbf{I}_{Q(L+1)} & 0
\end{array}\right], \\
& \mathbf{E}_{a, r, t}(k)=\left[\tilde{s}^{t T}(k) * \mathbf{B}(k) 0_{\mathrm{DQL} \times 1}\right],
\end{aligned}
$$

The channel $\mathbf{h}_{a, r, t}(k)$ and is denoted as,

$$
\mathbf{G}_{a}:=\left[\boldsymbol{I}_{Q(L+1)} 0_{Q(L+1) \times D Q(L+1)}\right] .
$$

$$
\begin{gathered}
\bar{K}(k)=\bar{P}(k \mid k-1) \boldsymbol{E}_{a, r, t}^{H}(k) \times\left[\mathbf{E}_{a, r, t}(k) \bar{P}(k \mid k-1) \mathbf{E}_{a, r, t}^{H}(k)+\sigma_{v}^{2}\right]^{-1} \\
\hat{\mathbf{h}}_{a, r, t}(k \mid k)=\hat{\mathbf{h}}_{a, r, t}(k \mid k-1)+\bar{K}(k) *\left[\boldsymbol{y}(k)-\mathbf{E}_{a, r, t}(k) * \hat{h}_{a, r, t}(k \mid k-1)\right] \\
\bar{P}(k \mid k)=\left[\boldsymbol{I}-\bar{K}(k) * \mathbf{E}_{a, r, t}(k)\right] * \bar{P}(k \mid k-1)
\end{gathered}
$$

We use $\hat{\mathbf{h}}_{a, r, t}(k)$ to predict $\hat{\mathbf{h}}_{r, t}(k)$ that is the complex-exponential basis expansion model coefficients,

$$
\hat{\bar{h}}_{r, t}(k)=\left\{\begin{array}{cc}
\hat{\bar{h}}_{a, r, t, D}(k+D) & k=1,2, \ldots, N_{E}-D \\
\hat{\bar{h}}_{a, r, t, N_{E^{-}}}(k+D) & k=N_{E}-D+1, \ldots, N_{E}
\end{array}\right.
$$

Based on (33), we can obtain

$$
\hat{\bar{g}}_{r, t}(k)=\mathbf{B}(k) * \hat{\bar{h}}_{r, t}(k)
$$

So we can obtain

$$
\hat{\bar{g}}_{r, t}(k)=\left\{\begin{array}{c}
E\left[\bar{g}_{r, t}(k) \mid \bar{y}(1), \ldots, \bar{y}(k+D)\right] \quad k=1,2, \ldots, N_{E}-D \\
E\left[\bar{g}_{r, t}(k) \mid \bar{y}(1), \ldots, \bar{y}\left(N_{E}\right)\right] \quad k=N_{E}-D+1, \ldots, N_{E}
\end{array}\right.
$$

Finally, we can have

$$
\widehat{\mathbf{H}}=\left[\begin{array}{ccc}
\hat{\bar{g}}_{1,1}(k) & \cdots & \hat{\bar{g}}_{1, n_{t}}(k) \\
\vdots & \ddots & \vdots \\
\hat{\bar{g}}_{n_{r}, 1}(k) & \cdots & \hat{\bar{g}}_{n_{r}, n_{t}}(k)
\end{array}\right]
$$

\section{Simulation Results}

The proposed detector is investigated through BER simulations. The system performances are evaluated in the 2 transmitted antennas and 2 received antennas configuration. The signal modulation is QPSK. The adopted channel model is the SCM channel model which is proposed in [23]. The simulations of our proposed iterative receiver are with 6 paths or with 14 paths. We use the sample input period as $2.4 \times 10^{-4}$. We assume the Doppler-frequency as $97 \mathrm{~Hz}$. The $T_{s}$ is $1 / 64$.

Table 1. Simulation environment in MIMO-OFDM SDFE

\begin{tabular}{|c|c|}
\hline Channel & Rayleigh, Rician, ITU(Rayleigh) \\
\hline Modulation & BPSK, QPSK \\
\hline FFT size & 64 \\
\hline CP length & 16 \\
\hline
\end{tabular}




\begin{tabular}{|c|c|}
\hline Iteration & $4,5,6$ \\
\hline Code rate & $1 / 2$ \\
\hline K-factor & $1 \mathrm{db}$ \\
\hline Antenna configuration & $2 \mathrm{X} 2$ \\
\hline velocity & $30,60 \mathrm{~km} / \mathrm{hr}$ \\
\hline
\end{tabular}

In order to make sure that the characteristic of the communication channels to be very fast fading, the $T_{s}$ and $T_{c}$ (coherence time) must be compared. The $T_{c}$ is the inverse of the Doppler frequency. $T_{c}=\frac{1}{97} \approx 0.01$. In the simulation, we choose the parameter $Q=9$ in the complex-exponential basis expansion model. $\mathrm{Q}$ must be bounded $\mathrm{Q} \geq 2\left[f_{d} T_{p} T_{s}\right]$ [3]-[7]. The parameter $\alpha$ that is used for the AR-1 channel model is selected as 0.996 . The Table 1 is the parameters of the simulation environment that are used in the simulations. The velocities we choose is 60 or $30 \mathrm{~km} / \mathrm{hr}$. We will compare the system performances of the proposed iterative receiver with those of the existing HDFE receiver structure and the existing MMSE-LE receiver structure. The Fig. 5 is the bit error rate system performances of the proposed iterative receiver, the LE and the HDFE in the ITU communication environment. 4 iterations are used in the simulation. The QPSK signal modulation is used. We find that the proposed iterative receiver outperforms the existing LE and HDFE methods. The proposed iterative recever is with very good channel impulse response estimation capability and can achieve very good bit error rate system performance.

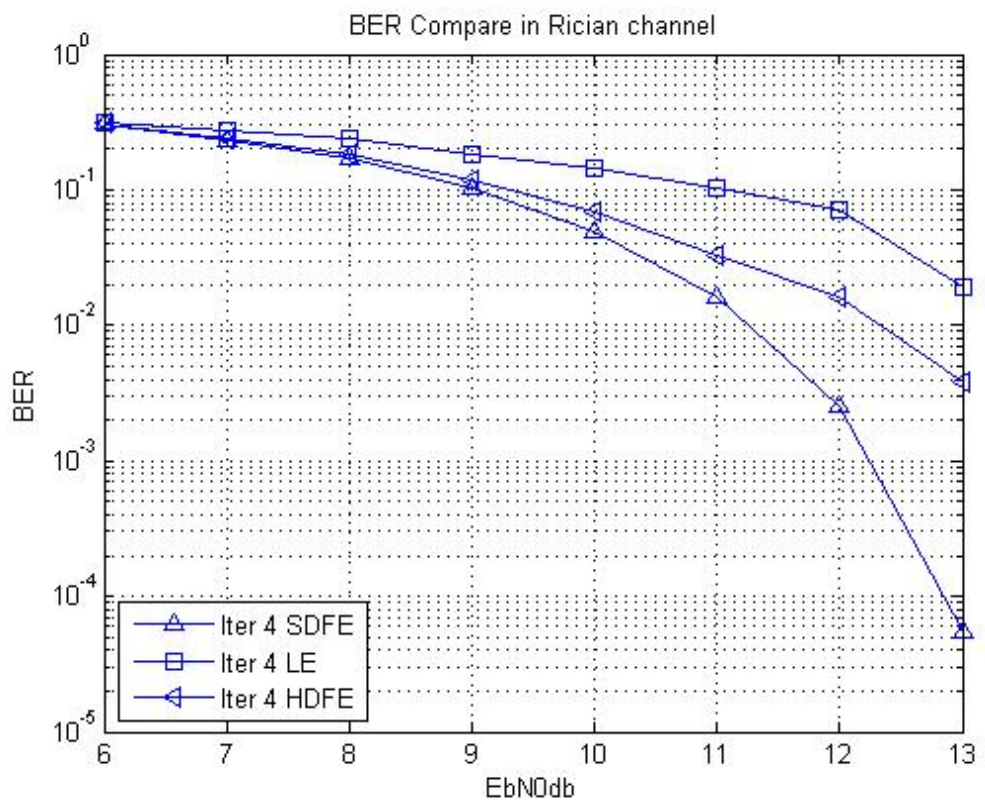

Fig. 5. The bit error rate performances of the iterative receiver, the LE and the HDFE in ITU communication. (The QPSK signal is used) 
The Fig. 6 is the bit error rate performances of the iteartive receiver with different iteration number in the Rayleigh communication. The BPSK modulation signal is used in the simulation. The Fig. 7 is the bit error rate performances of the proposed iterative receiver and those with the perfect channel state information and different iteration number in the Rayleigh communication. 4 and 3 iterations are used. The Fig. 8 is the bit error rate performances of the proposed iterative receiver with different iteration number in the ITU communication. The BPSK modulation signal is used. As compared with the case of the Rayleigh communication channel, we can see that the iterative receiver also converges at the 5th iteration.

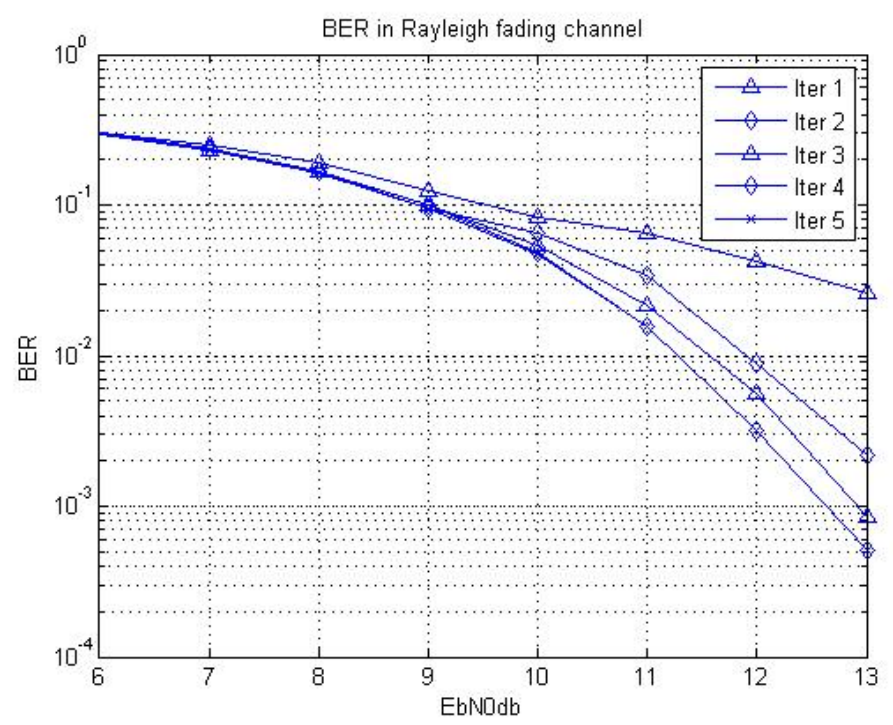

Fig. 6. The bit-error-rate performances of the proposed iterative receiver with different iteration number in the Rayleigh communication channel. (BPSK is used)

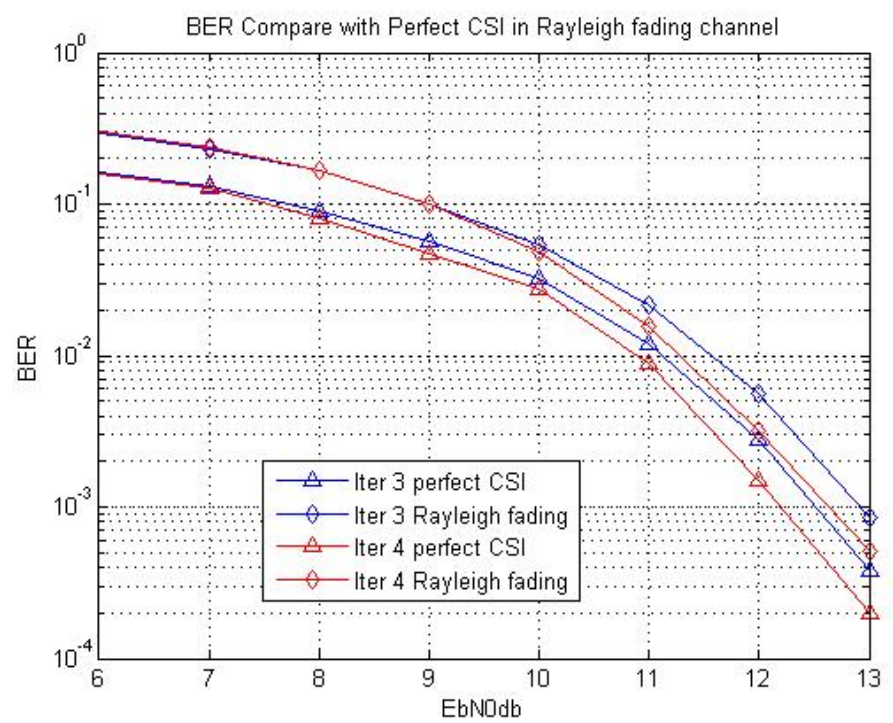

Fig. 7. The bit error rate performances of the iterative receiver and those with perfect channel state information and different iteration number in the Rayleigh communication. (The BPSK is used) 
The Fig. 9 is the bit error rate performances of the iteartive receiver and those with perfect channel state information and different iteration numbers in the ITU communication. The BPSK modulation is used. Compared with the case of the Rayleigh communication channel, we can find that the performance of the proposed iterative receiver approaches to that with ideal CSI.

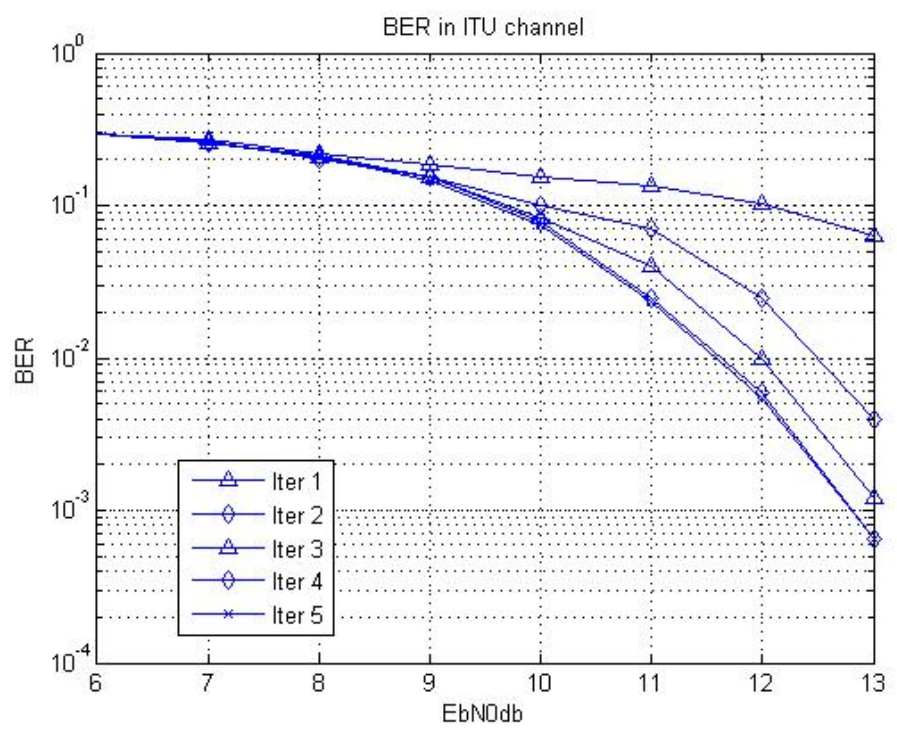

Fig. 8. The bit error rate performances of the iterative receiver with different iteration numbers in the ITU communication. (BPSK is used)

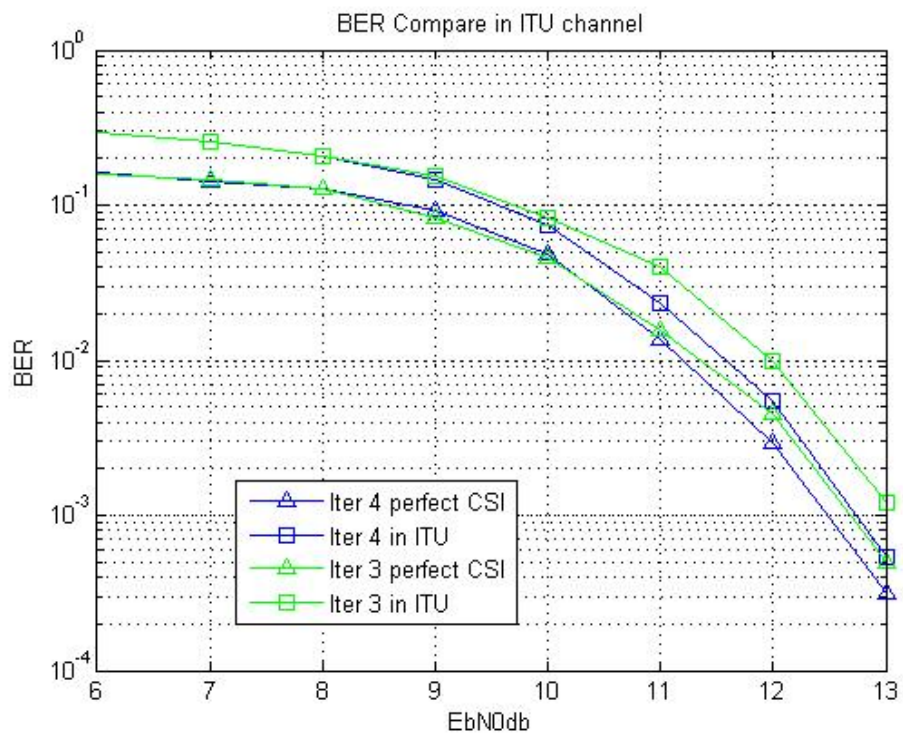

Fig. 9. The bit error rate performances of the iterative receiver and those with ideal channel state informantion and different iteration numbers in the ITU communication. (The BPSK is used)

The Fig. 10 is the bit error rate performances of the iteartive receiver with different iteration number in the Rician communication. The Fig. 11 is the bir error rate performances of the iterative receiver and that with ideal channel state information and different iteration 
numbers in the Rician communication channel.

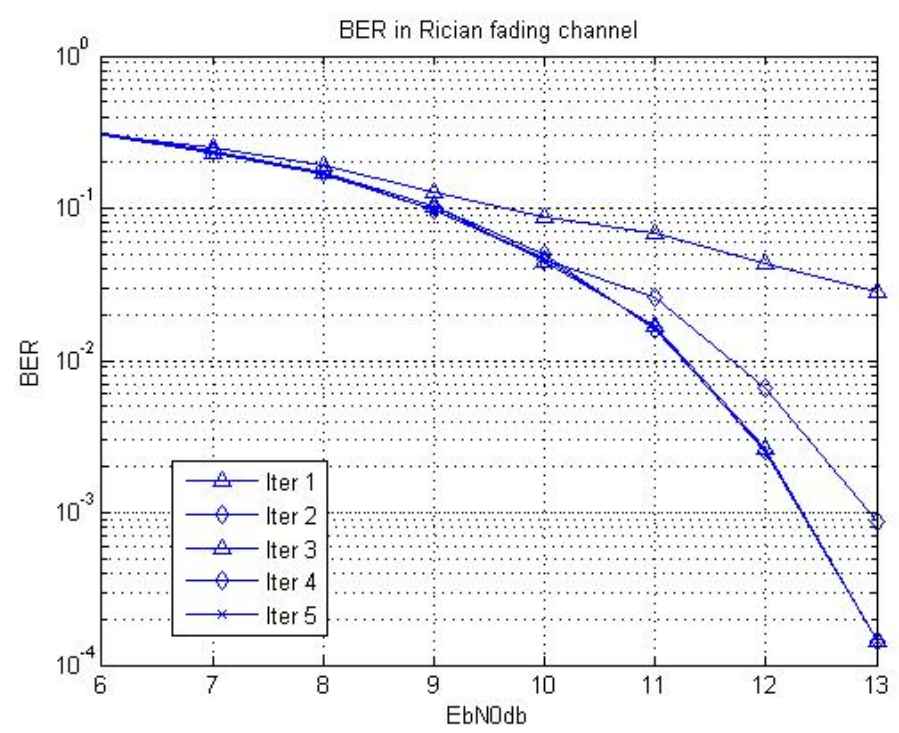

Fig. 10. The bit error rate performances of the iterative receiver with different iteration numbers in the Rician communication. (BPSK is used)

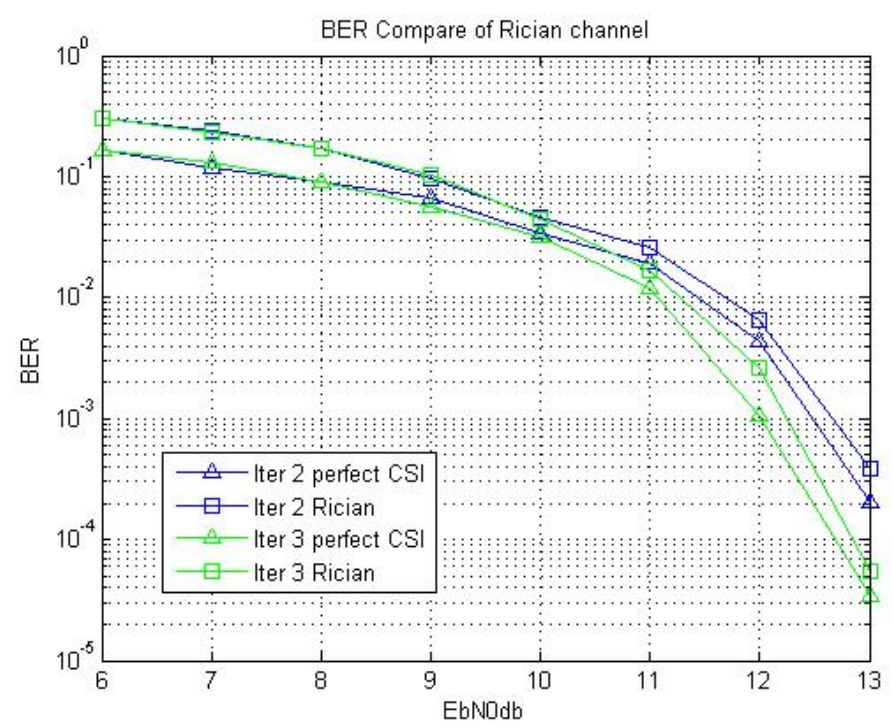

Fig. 11. The bit error rate performances of the iterative receiver and that with ideal channel state information and different iteration numbers in the Rician communication. (The BPSK is used)

The Fig. 12 is the bit-error-rate performances of the iterative receiver with different iteration numbers in the ITU cimmunication and with velocity $30 \mathrm{~km} / \mathrm{hr}$. The QPSK modulation is used. The iterative receiver can achieves very satisfactory system performance. The Fig. 13 is the bit-error-rate performances of the iterative receiver with different iteration number in the ITU communication and with velocity $60 \mathrm{~km} / \mathrm{hr}$. The QPSK modulation is used. We see that the iterative detector converges at the fifth iteration. The iterative receiver achieves very satisfactory system performance. 


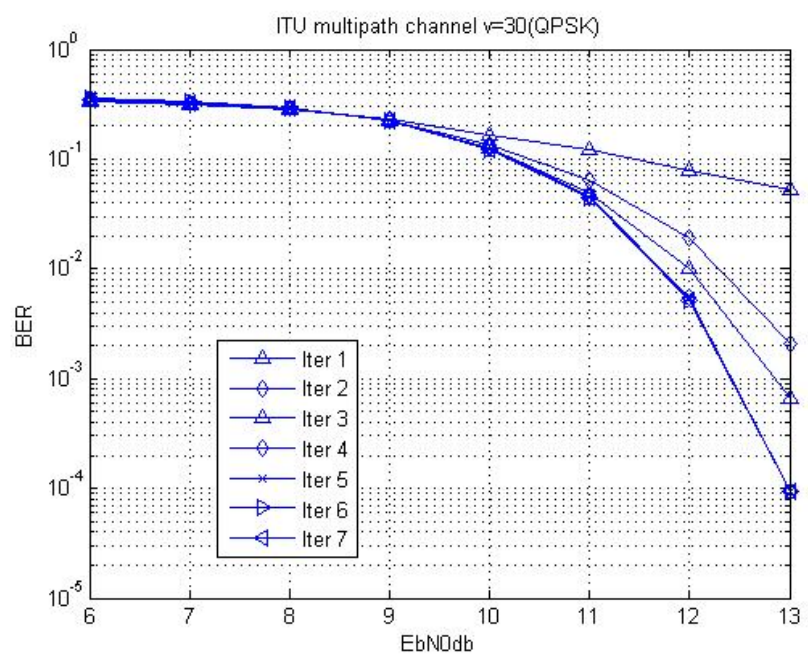

Fig. 12. The bit-error-rate performances of the iterative receiver with different iteration number in the ITU communication and velocity $30 \mathrm{~km} / \mathrm{hr}$. (QPSK signal is used)

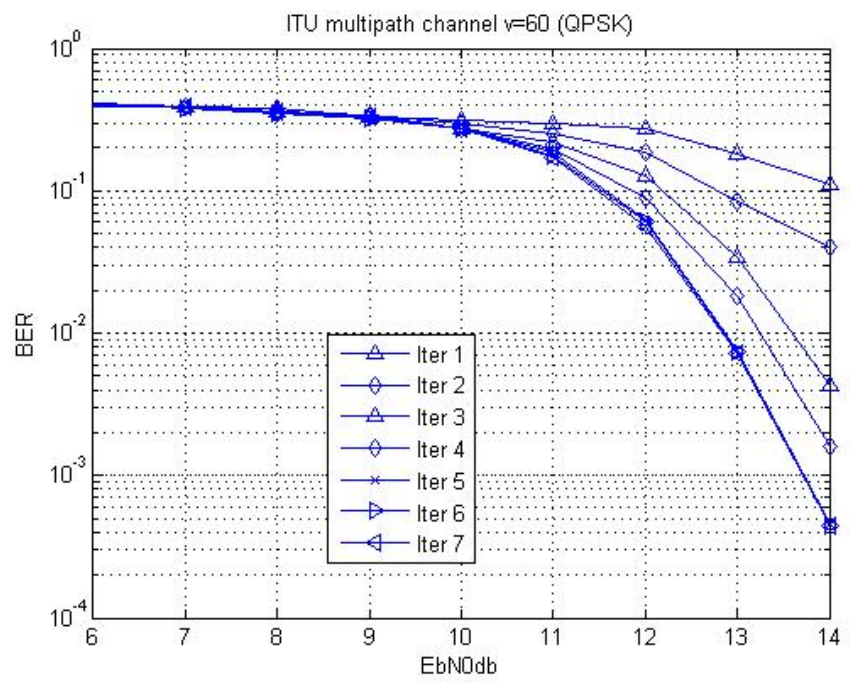

Fig. 13. The bit-error-rate performances of the iterative receiver with different iteration number in the ITU communication and velocity $60 \mathrm{~km} / \mathrm{hr}$. (QPSK signal is used)

\section{Conclusion}

This paper proposes a novel Kalman filter channel impulse response prediction and turbo channel equalizer for the MIMO-OFDM communication system in the fast multipath fading environments. The proposed iterative receiver can combats the error propagation effect in the fast multipath fading environment. This paper also compares the system performances of the iterative receiver with the existing receivers in several different fast multipath fading environments. 


\section{References}

[1] V. Namboodiri, H. Liu, and P. Spasojevi`c, "Low complexity turbo equalization for mobile MIMO OFDM systems,” in Proc. of International Conference on Communications and Signal Processing, pp. 255-260, Feb. 2011. Article (CrossRef Link)

[2] Movahedian, and M. McGuire, "Estimation of Fast-Fading Channels for Turbo Receivers With High-Order Modulation,” IEEE Transactions on Vehicular Technology, vol. 62, no. 2, pp. 667-678, Feb. 2013. Article (CrossRef Link)

[3] A. Movahedian, and M. McGuire, "Efficient and Accurate Semiblind Estimation of MIMO-OFDM Doubly-Selective Channels," in Proc. of IEEE Vehicular Technology Conference, pp. 1-5, Sept. 2014. Article (CrossRef Link)

[4] K. Muralidhar and D. Sreedhar, "Generalized vector state-scalar observation Kalman channel estimator for doubly-selective OFDM systems," in Proc. of IEEE International Conference on Acoustics, Speech and Signal Processing, pp. 4928-4932, May 2013. Article (CrossRef Link)

[5] A. Movahedian, and M. McGuire, "On the Capacity of Iteratively Estimated Channels Using LMMSE Estimators," IEEE Transactions on Vehicular Technology, vol. 64, no. 1, pp. 97-107, Jan. 2015. Article (CrossRef Link)

[6] X. Li and T. F. Wong, "Turbo equalization with nonlinear Kalman filtering for time-varying frequency-selective fading channels,” IEEE Transactions Wireless Communications, vol. 6, no. 2, pp. 691-700, Feb. 2007. Article (CrossRef Link)

[7] B. Chen, Q. Cui, F. Yang and J. Xu, “A Novel Channel Estimation Method Based on Kalman Filter Compressed Sensing for Time-Varying OFDM System,” in Proc. of Wireless Communications and Signal Processing, pp. 1-5, Oct. 2014. Article (CrossRef Link)

[8] W. Zhang, F. Gao and Q. Yin, "Blind Channel Estimation for MIMO-OFDM Systems With Low Order Signal Constellation,” IEEE Communications Letters, vol. 19, no. 3, pp. 499-502, Mar. 2015. Article (CrossRef Link)

[9] C. Douillard et al., "Iterative correction of intersymbol interference: Turbo-equalization," ETT, vol. 6, no. 5, pp. 507-511, Sep. 1995. Article (CrossRef Link)

[10] C. Berrou, A. Glavieux, and P. Thitimajshima, "Near Shannon limit error-correcting and decoding: turbo codes,” in Proc. of IEEE Int. Conf. Commun., vol.2, pp.1064-1070, May 1993. Article (CrossRef Link)

[11] S. Jeong and J. Moon, "Self-Iterating Soft Equalizer," IEEE Transactions on Communications, vol. 61, no. 9, pp. 3697-3709, Sept. 2013. Article (CrossRef Link)

[12] X. Yang, S. Houcke, C. Laot and H. Wang, "Soft Decision Feedback Equalizer for Channels with Low SNR in Underwater Acoustic Communications," in Proc. of IEEE OCEANS, pp. 1-5, June 2013. Article (CrossRef Link)

[13] J. Wu, L. Wang and C. Xiao, "Low complexity soft-interference cancellation turbo equalization for MIMO systems with multilevel modulations," in Proc. of IEEE Global Communications Conference, pp. 3353-3358, Dec. 2013. Article (CrossRef Link)

[14] C. Dong, J. Lin, K. Niu, Z. He and Z. Bie, "A Simplified Hard Decision Feedback Equalizer for Single Carrier Modulation with Cyclic Prefix," in Proc. of IEEE Vehicular Technology Conference, pp. 1-5, Sept. 2011. Article (CrossRef Link)

[15] C.-Y. Chung, F.-B. Ueng and Y.-K. Chang, "Frequency-Domain Iterative SDFE for MIMO-OFDM Systems,” Wireless Personal Communications, Vol. 86, Issue 3, pp. 1121-1140, Feb. 2016. Article (CrossRef Link)

[16] H. Viswanathan and M. Weldon, "The Past, Present, and Future of Mobile Communications," Bell Labs Technical Journal, vol. 19, pp. 8-21, Aug. 2014. Article (CrossRef Link)

[17] I. Barhumi, "Decision feedback turbo equalization for OFDM over doubly selective channel," in Proc. of IEEE Global Communications Conference, pp. 3801-3806, Dec. 2012. Article (CrossRef Link) 
[18] S. Ahmed, T. Ratnarajah, M. Sellathurai, and Colin F. N. Cowan, "Iterative Receivers for MIMO-OFDM and Their Convergence Behavior,” IEEE Transactions on Vehicular Technology, vol. 58, no. 1, pp. 461-468, Jan. 2009. Article (CrossRef Link)

[19] M. Tüchler, A. C. Singer, and R. Koetter, "Minimum mean square error equalization using a priori information,” IEEE Trans. Signal Process., vol. 50, no. 3, pp. 673-683, Mar. 2002. Article (CrossRef Link)

[20] A. Dejonghe and L. Vanderdorpe, "Turbo equalization for multilevel modulation: An efficient low-complexity scheme,” in Proc. of IEEE Int. Conf. Commun., vol. 3, pp. 1863-1867, 2002 Article (CrossRef Link)

[21] S. T. Brink, “Convergence behavior of iteratively decoded parallel concatenated codes," IEEE Transactions on Communications, vol. 49, no. 10, pp. 1727-1737, Oct. 2001. Article (CrossRef Link)

[22] H. Lou, C. Xiao and A. Rafati, "Low-Complexity Soft-Decision Feedback Turbo Equalization for MIMO Systems With Multilevel Modulations,” IEEE Trans. Vehicular Technology, vol. 60, no. 7, pp. 3218- 3227, Sep. 2011. Article (CrossRef Link)

[23] R. R. Lopes, Iterative estimation, equalization and decoding, Ph.D. dissertation, School of Electr. Comput. Eng., Georgia Inst. Technology, Atlanta, GA, 2003. Article (CrossRef Link)

[24] M. Tüchler, R. Koetter and A. C. Singer, “Turbo equalization: Principles and new results,” IEEE Trans. Commun., vol. 50, no. 5, pp. 754-767, May 2002. Article (CrossRef Link)

[25] P. Vila et al., Reduced-Complexity Soft Demapping for Turbo-Equalization, presented at the 2nd Int. Symp. Turbo Codes Related Topics, Brest, France, Sep. 2000. Article (CrossRef Link) 


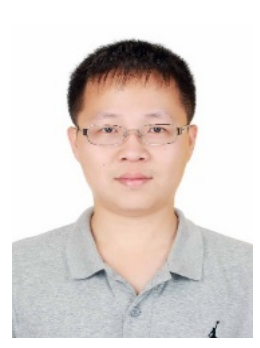

Yu-Kuan Chang was born in Kaohsiung, Taiwan, in 1986. He received the B.Sc. degree in aeronautical engineering from National Formosa University (NFU), Yunlin, Taiwan, in 2008 and the M.Sc. degree from the National Chi Nan University (NCNU), Nantou, Taiwan, in 2010. He is a member of the Communications System Laboratory, National Chung Hsing University (NCHU). His research interest is focused on MIMO MC-CDMA and LTE communication systems.

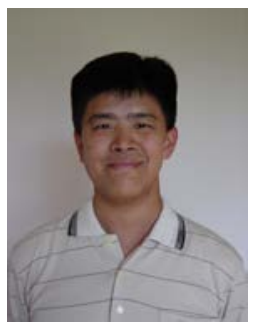

ang-Biau Ueng received the Ph.D. degree in electronic engineering from the National Chiao Tung University, Hsinchu, Taiwan in 1995. From 1996 to 2001 he was with National Space Program Office (NSPO) of Taiwan as an associate researcher. From 2001 to 2002 he was with Siemens Telecommunication Systems Limited (STSL), Taipei, Taiwan, where he was involved in the design of mobile communication systems. Since February 2002 he has been with the department of electrical engineering, National Chung-Hsing University, Taichung, Taiwan. His areas of research interest are wireless communication and adaptive signal processing.

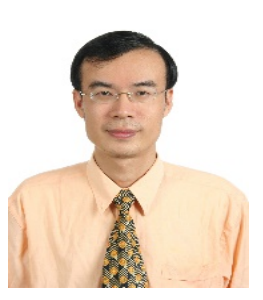

Ye-Shun Shen received the Ph.D degree in communication engineering from the National Cheng Kung University, Tainan, Taiwan, in 2004. From 1993 to 2005, he has been with Chunghwa Telecom, where he was a Senior Engineer. Since August 2005 he has been with the Department of Aeronautical Engineering, National Formosa University, Huwei Township, Yunlin county, Taiwan.His research interests include spread spectrum communications, wireless access technologies, and coding theory.

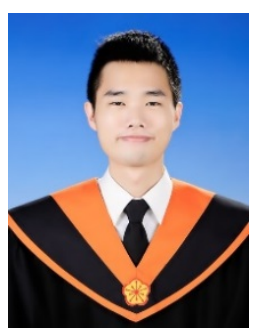

Chih-Yuan Liao was born in Taichung, Taiwan, on October 1989. He received the B.S. degree from the Department of Electrical Engineering, Chang Gung University (CGU), Linko, Taiwan, in 2013, and M.S. degree in communication engineering from the National Chung Hsing University (NCHU), Taichung, Taiwan, in 2015. He was a member of the Electronic Communication Systems Laboratory, NCHU. He is currently fulfilling his mandatory military service. His research interests include Long Term Evolution (LTE), multiple antenna communications, Orthogonal Frequency-Division Multiplexing, Soft Decision Feedback Equalizer, Kalman channel estimator, Fast-Fading channel and adaptive signal processing. 